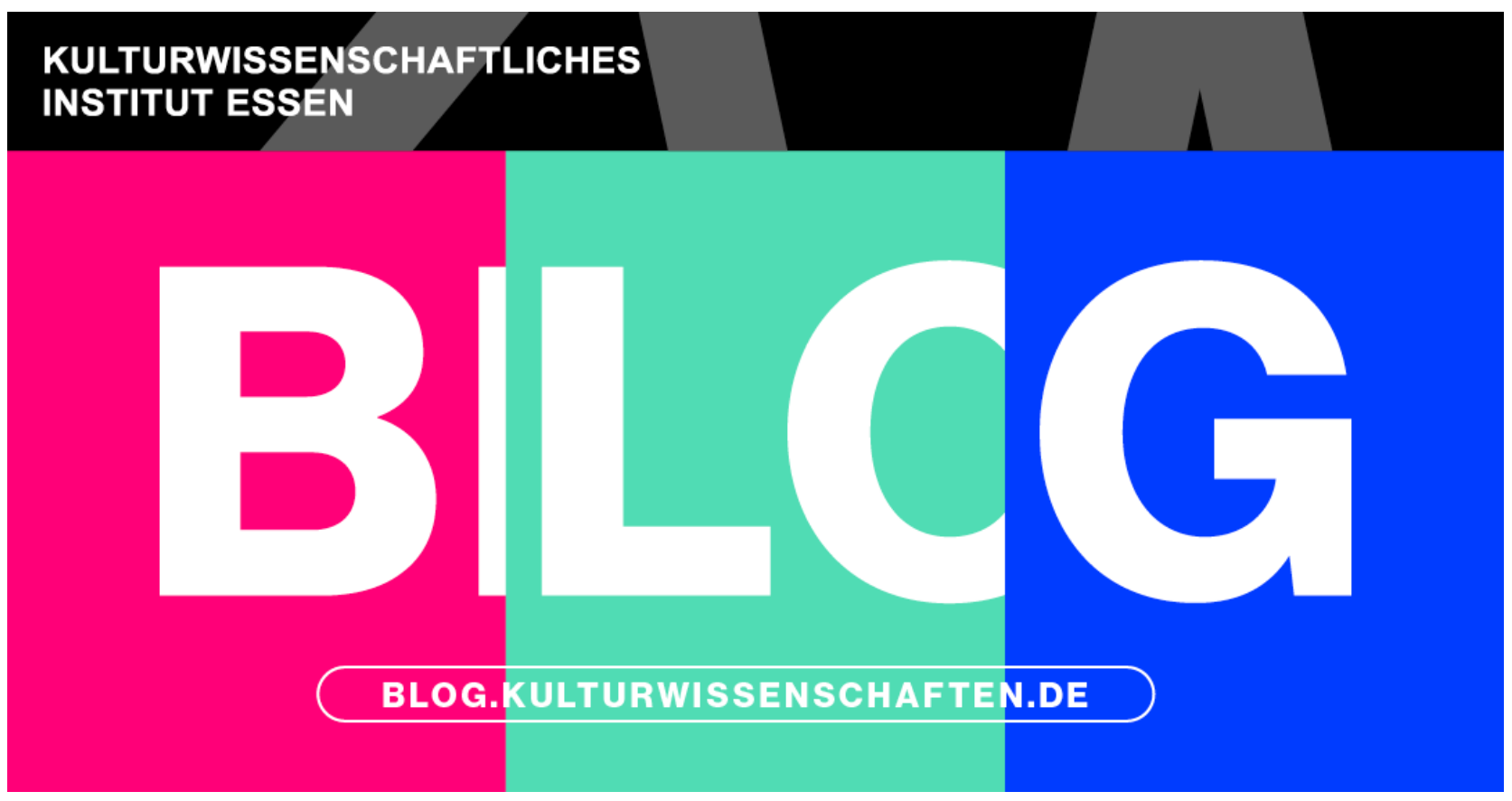

\title{
Kritik als Erklärvideo Expertise und Fiktion auf Youtube
}

Erschienen in: Populäre Expertise | Popular Expertise Von: Anja Schürmann

[Dieser Beitrag in der Reihe „Populäre Expertise" erscheint parallel auf dem KWI-Blog. und dem Blog_des SFB 1472 „Transformationen des Populären“.]

Ab wann ist man Experte? Ab dem Studienabschluss, der Ausbildung, ab zwanzig Jahren Berufserfahrung? Oder ab der Banderole, die im Video unten steht? Etymologisch ist der Experte jemand, der erprobtes, bewährtes Wissen zur Verfügung stellt. Der demnach das, was er machen soll, schon mal gemacht hat. Und das mit Erfolg, anders ließe sich bewährtes Wissen nicht herstellen.

Experten gibt es zuhauf in den Videos, die meinen Youtube-Algorithmus inzwischen irreparabel verschoben haben. Expert Reviews heißen sie bei Vanity Fair, Technique Critique bei Wired, The Breakdown bei GQ. Die Youtube-Playlist hält bei Vanity Fair 42 Clips mit im Durchschnitt 20 Minuten, bei Wired immerhin 33 ungefähr 25-minütige Clips und bei GQ 48 10- bis 40-minütige Videos vor. Um was geht es? Es geht um außer Kontrolle geratene Faktenchecks, eine journalistische Technik der redaktionellen Sichtung, die sich in diesen Fällen nicht mit der Verifizierung von Reportagen, sondern von fiktionalen Filmen und Serien beschäftigt. Die Expertise der Expert*innen ist dabei keineswegs rein akademisch: Eine ehemalige Olympionikin beschäftigt sich mit der Darstellung von Olympia im Film, ein Betrugsexperte in Casinos musste sehr viele 
Steven Soderbergh-Filme gucken und der feuchte Preppertraum Bear Grylls bewertet, ob in Filmen, in denen das Überleben der Protagonist*innen im Vordergrund steht, auch wirklich angemessen überlebt wurde.

Bleiben wir doch gleich bei dieser Folge. Natürlich befindet sich der Überlebensexperte Bear Grylls nicht wie seine Kolleg*innen im Studio, sondern schaukelt vor Blau, ist also draußen, vielleicht sogar in der ,Wildnis‘. Motivisch passend befinden sich die Schiffbrüchigen im ersten gezeigten Film The Life of Pi ebenfalls auf Blau, die „Survival Conditions“ sind in Spiegelstrichen eingeblendet: „- stranded at Sea, - no food, - no fresh water". Ob das was werden kann, liebe Tigerente? Gezeigt wird ein Fischfang, bei dem Pi Patel erst mal dafür kritisiert wird, überhaupt essen zu wollen. Trinken sei viel wichtiger! Abgeschlossen wird die gründliche einminütige Analyse durch eine Tabelle, die mit „How Realistic?“ überschrieben ist. Life of Pi darf sich in erlaubtem Grün das Prädikat „realistic“ abholen, bevor der nächste Film drankommt. Am Ende steht es 4:2, nur zweimal musste die rote Karte „not realistic“ in der rechten Spalte vergeben werden. In All is Lost ist Robert Redfords Signalfeuer zu schnell außer Kontrolle geraten und der Wolf, mit dem Liam Neeson in The Grey konfrontiert ist, würde eigentlich nie von sich aus und ebenfalls nie alleine angreifen (Abb. 1). Bear Grylls gibt sich im Schnitt zufrieden und lobt die Filmschaffenden, inzwischen mehr Recherche zu betreiben, um realistischere Szenarien zu kreieren.

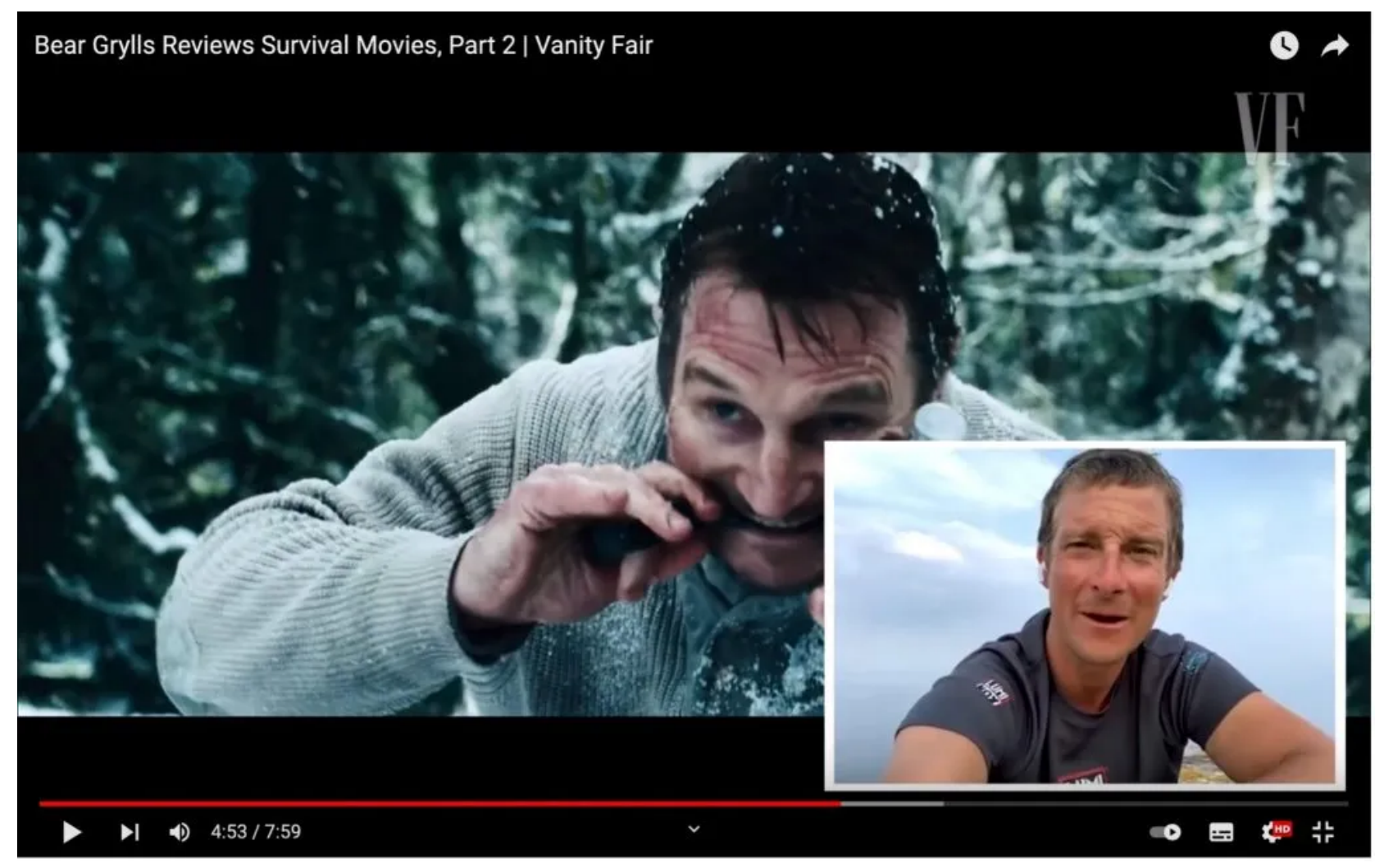

Abb. 1: Screenshot aus Bear Grylls Reviews Survival Movies, Part 2, Vanity Fair, https://youtu.be/f5LCJx8LqTI, abgerufen am 4.1.2022.

Bei Les Stroud oder dem „Survivor Man“ in der halbstündigen GQ-Episode The Breakdown bleibt es nicht bei der Einschätzung einzelner Szenen. Stroud wundert sich über den Ausgang ganzer Filme. Into the Wild hätte nie so enden müssen, der Tod von 
Christopher McCandless wäre vermeidbar gewesen, wenn seine survival skills besser gewesen und sein Elch besser konserviert worden wäre. Niemand muss in Alaska verhungern. Bei Into the Wild handelt es sich um eine Verfilmung nach einer wahren Gegebenheit: Christopher McCandless ist wirklich in Alaska verhungert, die literarische Vorlage für den Film stammt bekanntlich aus der Tastatur Jon Krakauers, der erst einen Artikel und dann ein Buch gleichen Titels über McCandless schrieb. Was die Aussage im Video nicht nur pietätlos, sondern auch ziemlich zynisch erscheinen lässt: Schade, dass McCandless keinen Überlebenskurs bei Stroud gebucht hatte. Er hätte es schaffen können.

Bei diesen beiden Videos wird ein Duell Experte vs. Experte inszeniert, einen sieht man, der oder die andere ist unsichtbar: Im Produktionsprozess kaufen sich die hier verhandelten Filme, die man ausnahmslos großen, finanzstarken Studios verdankt, Expertise ein. Es gibt Berater auf vielen Ebenen: Kürzlich hat der sog. ,Intimacy Coach“ Schlagzeilen gemacht, weil er Sexszenen aushandelte. ${ }^{1}$ Diesem unsichtbaren Experten, der allenfalls als Credit schnell über die Leinwand gescrollt wurde, wird hier seine Expertise vom sichtbaren Experten entweder gezollt oder abgesprochen, wobei die Videokritik leicht zu einem Bewerbungsfilm für kommende Produktionen werden kann.

Das ist nicht immer so. Im zweiten Video von Vanity Fair hat man wieder vor weißer Studiokulisse Platz genommen. „I was the food stylist from Julie \& Julia“ sind die ersten Sätze von Susan Spungan, sie ist somit als Expertin legitimiert. Und tatsächlich hat sie bei vielen Filmen, die in dem fünfzehnminütigen Video gezeigt werden, selbst Hand angelegt. Keine Konkurrenz wird hier etabliert, es wird weniger bewertet denn erläutert: Spungan erzählt der geneigten Zuschauerschaft, dass in einem Film mit an Sicherheit grenzender Wahrscheinlichkeit nie Eis gegessen wird: Zu heiße Scheinwerfer, zu viele Einstellungen und somit Schmelzzeit, weshalb Eis von Food Stylisten ,nachgebaut" werden muss. Interessant sind auch Szenen von nichteinvernehmlichem Essen: Hier führen schnelle Schnitte zwischen Nahaufnahmen vom Essen und dem späteren Kauen dazu, dass die tatsächliche Nahrungsaufnahme ungezeigt bleibt und die Schauspieler nicht allzu viel von was auch immer im Mund haben müssen. Das Video gleicht einem behind the scenes-Studiorundgang mit einer kompetenten Führung, die auch Filme, in denen Aussage und Filmbild nicht unbedingt harmonisieren, dafür entschuldigt, weil auch das eine Aussage ist: Realismus ist nur dann vonnöten, wenn Realismus Darstellungsziel ist. Nicht so bei Emily in Paris (Min. 3), deren zu blutiges Steak gar nicht gezeigt werden muss, um den attraktiven Koch aus der Küche zu locken.

Als Zuschauer*in habe ich die Wahl: Ich kann diese Videos entweder als Rezension oder als Bonusmaterial betrachten. Als Bonusmaterial stellen sie - wie im Fall von Susan Spungan - ein im Film oft unmarkiertes Gewerk vor, das aus seiner Perspektive, und ja, auch Expertise, das eigene Tun und seine Rolle im filmischen Produktionsprozess erläutert. Eine Erläuterung ist eigentlich die ureigenste Textsorte des Experten, da hier „das Gesehene als Anlass für die Darstellung von (Vor-)Wissen und (Aus-)bildung“ begriffen wird. ${ }^{2}$ In Erläuterungen gibt es immer eine epistemische Anreicherung, Wissen wird angefügt, Wissen, das oft als Grundlage für weitere Operationen wie die Deutung und Bewertung gesehen wird und beide argumentativ festigt und legitimiert. 
Deuten und Bewerten sind klassische Textsorten der Rezension, wobei diese hier die Einseitigkeit ihrer Lesart kaum verstecken können. Kein Rezensent beschäftigt sich nur mit den ersten zehn Minuten eines Films, daher muss ich einen Politthriller auch nicht nur ausschließlich aus der Perspektive der Politikberatung goutieren oder einen Film, in dem überwiegend überlebt wird, aus der Perspektive eines Survival-Trainers. Außerdem haken diese Rezensionen bei der Fiktion nach. Expertenvideos sehen Filme nicht als fiktive Werke, deren fiktiven Vereinbarungen es zu folgen gilt. Der Fiktionsvertrag liegt hier unterschriftsreif auf dem Tisch, doch der Stift zickt, da mir der Experte sagt, dass man Fische nicht so zubereiten sollte, wie es Tom Hanks in Cast Away gemacht hat.

Versagt hier retrospektiv die Fiktion, weil der Film seinen realistischen Erzählduktus mit einer Erwartung angereichert hatte, die nun als Konstruktion - oder, wahrscheinlicher, als schlampige Recherche - sichtbar wird? Ja, aber nicht nur. Ein Film kann alles richtig, aber falsch machen. Klar, Sandra Bullock hätte nie so schnell in eine andere Richtung geschleudert werden können, wie in Gravity gezeigt und vom Astronauten Chris Hadfield bemängelt. Was Astronaut Chris Hadfield aber nicht berücksichtigt, sind die visuellen und damit ästhetischen Folgen dieses Unfalls. Die Folgen, für die man vielleicht einen Fiktionsexperten braucht, der dem Weltraumexperten Paroli bietet.

Ich probiere es mal: Ja, manche Dinge sind so falsch und traurig, dass jede Frage an sie unbeantwortet bleibt. Das ist auch bei Filmen so. Allerdings sind sie nicht notwendigerweise schlecht, weil sie falsch sind. Das Spiel, auf das sich Kendall Walton in seiner Make-Believe-Theorie bezieht, beruht nur bedingt auf Wissen, ganz wesentlich aber auf Glauben. Dieser Glaube muss plausibilisiert sein, allerdings darf seine Plausibilität nie vollständig sein, sonst müsste man ja nicht glauben. Diesem Glauben entspricht etwas, was Walton die fiktive Wahrheit nennt, ${ }^{3}$ eine Wahrheit, die in einer Fiktion - einem Film - eine andere Rolle spielt als die wissenschaftliche Wahrheit, auf die sich der Astronaut Chris Hadfield bezieht. Jene fiktive Wahrheit ist dafür zuständig, dass wir Godzillafilme als Fiktionen und nicht als unwahren Quatsch betrachten können, aber sie macht auch vor Filmen nicht halt, die ein mimetischeres Verhältnis zur sogenannten Wirklichkeit haben. Bleiben wir bei Gravity. Sandra Bullock ist also inkorrekterweise auf einem Weltraumspaziergang recht schnell und in die falsche Richtung geschleudert worden. Die dramaturgischen Gründe hierfür liegen auf der Hand: Ein schwerelos langsamer und behutsamer Außeneinsatz wird dadurch dramatisiert, wird schneller und im narrativen Bogen des Films ,problematischer‘. Daher kann in dieser ,Unwahrheit' ein „Fiktionssignal[] auf der Ebene der Erzählung"4 gesehen werden: Die durch die fliegende Kamera, wo auch immer sie im Weltraum befestigt sein soll, Schnitt und Musik zusätzlich als dramatisch akzentuierte Szene macht auch durch ihre multimodale Anreicherung deutlich, dass man sich in einer Fiktion befindet. Mein Stolpern im Treppenhaus ist selten mit Musik unterlegt. Gleichzeitig gibt es eine narrative Logik: Sandra Bullock muss schwer erreichbar für George Clooney (dem anderen Astronauten auf Weltraumspaziergang) sein, um den weiteren Handlungsverlauf zu plausibilisieren. Dass diese schwere Erreichbarkeit nun maximal dramatisch in Szene gesetzt wird, wobei auf „physikalische Gesetze“, wie der Astronaut Chris Hadfield anmerkt, nicht besonders eingegangen wird, mag auch an etwas liegen, was die Fiktionstheoretikerin Marie-Laure 
Ryan „Zugänglichkeitsrelationen“ ${ }^{5}$ genannt hat. Das All und seine Gesetze der Trägheit sind uns nicht ganz so bewusst wie andere Dinge. Deshalb können sie auch nicht ganz so leicht als falsch identifiziert werden.

Das war nur ein Fußnagel in der Tür möglicher Nutzungen fiktionaler Theorien für die Rezeption, dieses Blogformat von 9000 Zeichen erlaubt kaum mehr. Aber sollte mir am Ende ein Wunsch erlaubt sein, bin ich stark dafür, dass man in die weiße Studiokulisse neben Akzentexperten, Tätowierern, Kletterern und - überproportional häufig ehemaligen FBI-Agenten bald mal jemanden mit Fiktionskompetenz einlädt.

\section{References}

1. https://www.theguardian.com/tv-and-radio/2019/oct/06/safe-sex-on-screenintimacy-coordinators, abgerufen am 4.1.2022.

2. Hausendorf, Heiko (2004): Bildbeschreibungen zwischen Wahrnehmung und Erwartung. Linguistische Facetten einer interdisziplinären Thematik (Norm und Symbol. Die kulturelle Dimension sozialer und politischer Integration 52), Konstanz: Universität Kulturwissenschaftliches Forschungskolleg, 2004, S. 6.

3. Walton, Kendall L. (1990): Mimesis as Make-Believe. On the Foundations of the Representational Arts, Cambridge: Harvard University Press, 1990, S. 69. Es gibt zahllose andere Fiktionstheorien. Waltons ist eine der prominenteren, an der sich die Forschung immer noch abarbeitet.

4. Zipfel, Frank (2014): 5. Fiktionssignale, in: Tilmann Köppe und Tobias Klauk (Hg.), Fiktionalität: Ein Interdisziplinäres Handbuch, Berlin/New York: De Gruyter, 2014, S. 97-124, hier S. 110.

5. Ryan, Marie-Laure (1991): Possible Worlds, Artificial Intelligence, and Narrative Theory, Bloomington: Indiana University Press, 1991, S. 24.

SUGGESTED CITATION: Schürmann, Anja: Kritik als Erklärvideo. Expertise und Fiktion auf Youtube, in: KWI-BLOG, [https://blog.kulturwissenschaften.de/kritik-als-erklarvideo/], 24.01.2022

DOI: https://doi.org/10.37189/kwi-blog/20220124-0830 


\section{DuEPublico}

Dieser Text wird via DuEPublico, dem Dokumenten- und Publikationsserver der Universität Duisburg-Essen, zur Verfügung gestellt. Die hier veröffentlichte Version der E-Publikation kann von einer eventuell ebenfalls veröffentlichten Verlagsversion abweichen.

DOI: $\quad 10.37189 / \mathrm{kwi}-\mathrm{blog} / 20220124-0830$

URN: urn:nbn:de:hbz:464-20220124-183012-9 